\title{
Y a pesar de todo seguimos ahumando a los demás.
}

\author{
Jordi Pericàs Beltrán("); Concepción Zaforteza Lallemand ${ }^{(2)}$ \\ (1) Universitat de les Illes Balears. Profesor titular del Departament d'Infermeria i Fisioteràpia. \\ (2) Universitat de les Illes Balears. Profesora ayudante del Departament d'Infermeria i Fisioteràpia.
}

Enviar correspondencia a:

Dr. Jordi Pericàs Beltrán. Universitat de les Illes Balears. Departament d'Infermeria i Fisioteràpia. Carretera de Valldemossa Km. 7,5.

Edificio Guillem Cifre de Colonya. 07122-Palma de Mallorca. Teléfono: 971 173306. Telefax: 971 173190. E-mail: jordi.pericas@uib.es

Recibido: 21 de noviembre de 2003.

Aceptado: 3 de marzo de 2004.

\section{RESUMEN}

Objetivos: Un edificio universitario en Palma de Mallorca (España) fue declarado "libre de humo de tabaco" en octubre de 2002. En este estudio se ha entrevistado una muestra de usuarios hallados fumando en zonas prohibidas, evaluándose el conocimiento de la prohibición de fumar, la consciencia de estar perjudicando y molestando a las personas que comparten el mismo espacio y qué justificación daban a su conducta tabáquica.

Métodos: Estudio descriptivo transversal, mediante encuesta verbal con un cuestionario que consta de 6 preguntas cerradas y una abierta. Se ha tomado una muestra de 50 sujetos que han sido hallados fumando en zonas prohibidas.

Resultados y Conclusiones: Casi la totalidad de los encuestados (98\%) tienen conocimiento de la prohibición de fumar donde lo hacen. El $80 \%$ conoce la existencia de zonas para fumadores. Una amplia mayoría cree que el tabaco perjudica y molesta a los fumadores pasivos (98\% y 94\%). Uno de cada cinco opina que no deben restringirse las zonas donde se pueda fumar. Los sujetos que fuman en los lugares no permitidos son perfectamente conscientes de la prohibición, conocen los espacios destinados a fumadores y reconocen perjudicar y molestar a los fumadores involuntarios. De estos conocimientos no se desprende una conducta respetuosa sino que incluso se reclama el derecho a fumar en los espacios compartidos. Ningún encuestado afronta su responsabilidad personal ni reconoce su adicción como motivo de su conducta.

Palabras clave: Hábito tabáquico. Promoción de la salud. Tabaquismo pasivo. Universidad. Adicción

\section{ABSTRACT}

Aims: An university building in Palma de Mallorca (Spain) was declared "smoke free" in October 2002. In this study we interviewed a sample of users found smoking in prohibited areas, evaluating their knowledge of the prohibition on smoking, the awareness of harming and disturbing the people who share the same space and what justification they gave for their smoking behaviour.

Methods: Transversal descriptive study, by a verbal survey with a questionnaire consisting of 6 closed questions and one open one. A sample of comprised 50 subjects found smoking in prohibited areas.

Results and Conclusions: Practically all (98\%) were aware of the prohibition on smoking in the area where they did so. $80 \%$ of those surveyed knew of the existence of designated smoking areas. A large majority believe smoking harms and disturbs passive smokers (98\% and 94\%). One in five is of the opinion that smoking should not be restricted to certain areas. The subjects who smoke in the areas where it is not allowed are perfectly aware of the prohibition, they know the areas designated for smoking and acknowledge harming and disturbing involuntary smokers. The outcome of this knowledge is not respectful behaviour, but rather a claim to the right to smoke in shared area. Not one of those surveyed accepts his or her personal responsibility or recognises their addiction as a motive for their behaviour.

Key words: Smoking habit. Health promotion. Passive smoking. University. Addiction. 


\section{INTRODUCCIÓN}

Y a en 1964, el Servicio de Salud Pública de los Estados Unidos editó un informe que ponía de manifiesto algunos efectos perjudiciales del tabaco. En 1992 la Agencia de Protección del Medio Ambiente de los Estados Unidos (EPA), emitió un nuevo informe, sumamente revelador, acerca de los efectos del humo del tabaco sobre la salud de los fumadores pasivos ${ }^{2} y$ ha sido objeto de controversia por parte de la industria tabaquera ${ }^{3}$. En 1993, la EPA lanzó un comunicado de prensa en el que se catalogaba el humo del tabaco como un carcinógeno de clase A (la categoría que para la EPA indica la máxima certeza científica de que una sustancia es carcinógena y que no tiene concentraciones mínimas por debajo de las cuales se pueda considerar segura) ${ }^{4}$. En nuestros días, casi nadie discute -salvo que tenga dudosos intereses en ello- que el humo del tabaco es perjudicial para la salud, tanto de los fumadores activos como pasivos ${ }^{6}$. Así lo han reconocido la Organización Mundial de la Salud 6,7, y los distintos estados que la componen, incluido el Reino de España ${ }^{8}$. Todo ello se ha traducido en normas -más o menos tenidas en cuenta- que regulan el consumo, venta y publicidad de tabaco ${ }^{9,12}$.

En nuestro país, desde 1986, la ley General de Sanidad considera el aire contaminado por humo de tabaco "sustancia nociva para la salud de la persona"9. Se ha estimado que cada año, mueren al menos 55.000 personas por patologías asociadas al tabaquismo activo y más de 700 personas por patologías relacionadas con el tabaquismo pasivo, siendo las más frecuentes el cáncer de pulmón, las enfermedades cardiovasculares y los trastornos respiratorios ${ }^{13}$.

Aún constituyendo un hecho cotidiano y general, las relaciones entre fumadores activos y pasivos son un tema que ha sido poco estudiado y del que se ha escrito poco. Desgraciadamente, es por todos conocida la reiterada violación del derecho de los no fumadores a respirar aire libre de humo de tabaco llevada a cabo por los fumadores en los espacios públicos. A pesar de esta constatación y del amparo legal que -en caso de litigio- los fumadores poseen desde 1988, la ley ha sido sistemáticamente incumplida. Baste para ello echar una ojeada a cualquier hospital, centro sanitario, centro docente, cines, aeropuertos ${ }^{14} \ldots$

Sociológicamente, existen dos posicionamientos claros respecto al problema: por una parte la comunidad médica y científica que aboga por denunciar los efectos perniciosos del tabaquismo activo o pasivo sobre la salud y que ha dado fundamento para la promulgación de leyes y normas que hablan de limitar el hábito de fumar en los espacios públicos ${ }^{6-11}$ y por la otra parte, la industria tabaquera y los grupos que defienden los derechos de los fumadores a hacerlo en los espacios públicos y que a través de la publicidad (principalmente dirigida a mujeres ${ }^{15}$ y niños ${ }^{16,19}$ ) nos quieren inculcar que fumar "es normal" cuando no atractivo, elegante o atrevido ${ }^{20} \mathrm{o}$ incluso minimizar $\mathrm{o}$ falsear los efectos nocivos del humo del tabaco ${ }^{3}$.

Se han realizado algunos interesantes estudios como el de Polland ${ }^{21}$ en Canadá, que ilustra las relaciones entre fumadores y no fumadores en los espacios públicos, analizando lo que cada una de las partes reconoce como consideración o desconsideración respecto de la otra en el hábito de fumar.

En general, la asertividad de los no fumadores frente a los fumadores que contaminan el aire compartido es muy débil22. Así en España desde que entró en vigor el Real Decreto 192/1988 ${ }^{10}$ que daba sistemáticamente la razón a los no fumadores sobre los fumadores en caso de conflicto no se ha producido ni una sola denuncia.

En el Departamento de Enfermería y Fisioterapia de la Universitat de les Illes Balears, adhiriéndonos a la corriente internacional en defensa del derecho a respirar aire libre de humo de tabaco de los fumadores pasivos o involuntarios, en 2001 propusimos a los usuarios de nuestro edificio la declaración del mismo como "libre de humo de tabaco". En el edificio Guillem Cifre de Colonya, -situado en el Campus de la Universitat de les Illes Balears (España)- desarrollan sus actividades académicas más de 4500 alumnos, más de 200 profesores y trabajan en administración y servicios más de 30 personas. En él se ubican las Facultades de Psicología, Ciencias de Educación y la Escuela de Enfermería y Fisioterapia. Se propuso este edificio como piloto "libre de humo de tabaco" en la Universidad debido a que los estudios que se imparten en él pueden merecer la consideración de modélicos en cuanto a la educación para la salud ${ }^{23}$.

Una vez consensuada dicha declaración se llevaron a cabo una serie de acciones dirigidas a informar y concienciar sobre la prohibición de fumar en los espacios compartidos.

A pesar de todas las medidas adoptadas: conferencias, señalización de zonas compartidas y de zonas donde se permite fumar, folletos informativos, oferta gratuita de cursos de deshabituación, etc. todavía es frecuente ver personas fumando en áreas prohibidas.

A una muestra de estas personas se le ha pasado un sencillo cuestionario con el objeto de conocer su grado de información sobre la prohibición de fumar en los espacios compartidos, indagar cómo justificaban su conducta y evidenciar su ilegalidad. En este trabajo se publican y analizan los resultados de dicha encuesta. Éste ha sido un paso previo para fundamentar un estudio cualitativo cuyo objetivo será analizar las actitudes de los fumadores que no respetan las zonas delimitadas para hacerlo y la de los fumadores pasivos frente a este hecho. 


\section{SUJETOSY MÉTODOS}

El diseño elegido para realizar este estudio es descriptivo transversal, mediante una encuesta verbal que consta de 6 preguntas cerradas y una cuestión abierta. Las cuestiones que se plantearon pueden verse en la tabla 1.

La población a estudio son los usuarios que fuman en lugares prohibidos del Edificio Guillem Cifre de Colonya. La muestra se compone de los 50 primeros que fueron hallados durante los días 8 a 17 de octubre de 2003, ya que a partir de este número se "saturaron" los datos, es decir se mantenían las proporciones, siendo cada vez mas frecuente volverse a encontrar con los mismos entrevistados que fumaban en días anteriores.

Antes de la recogida de información se llevó a cabo una encuesta piloto sobre el cuestionario a un grupo de 10 personas y se corrigieron aquellos items en los que no estaban claros los planteamientos.

La estrategia de recogida de información, se organizó de acuerdo a la accesibilidad de los encuestados, estableciendo un máximo de 7 cada día para diversificar las situaciones.

Los datos fueron transcritos manualmente a una hoja de cálculo, para posteriormente ser analizados con el paquete estadístico SPSS v.11.5. Se estimaron proporciones y medias con su intérvalo de confianza al $95 \%$. En los intervalos de confianza se ha calculado el intervalo exacto.

Simultáneamente a la recogida de datos se llevó a cabo un diario de incidencias, en el que se anotaban aquellos aspectos que pudieran modular el resultado de la encuesta, ya fuera la hora del día, el periodo entre determinadas clases, la actitud de los encuestados y sus opiniones fuera de la encuesta.

\section{RESULTADOS}

Fueron entrevistados un total de 50 personas, 48 alumnos y 2 trabajadores del personal de administración y servicios. El $82 \%$ de la muestra son mujeres y el $18 \%$ hombres.

A través de un estudio paralelo, referido a la población total del edificio, sabemos que su media de edad es de 22,23 años (IC 95\% 21,82, 22,64) y que la prevalencia global de fumadores habituales y ocasionales es del $35 \%$ ( $37 \%$ en mujeres y $24 \%$ en hombres).

La totalidad de sujetos invitados a responder al cuestionario aceptaron la propuesta.

El conjunto de resultados, con un intervalo de confianza del 95\%, puede verse en la tabla 1.

Un quinto de los encuestados declara no saber que está prohibido fumar donde el sujeto lo hace, en tanto que el resto sí lo sabe. En la cuestión 2, sólo un sexto afirma no saber de la existencia de espacios destinados a fumadores.

Cuatro quintas partes de los encuestados declara no saber que la decisión de declarar el edificio "libre de tabaco" ha sido consensuada y aprobada por mayoría absoluta por alumnos, profesores y personal de administración y servicios en juntas de facultad o escuela de los estudios que se imparten en el edificio. El $22 \%$ afirma conocer este extremo.

En cuanto a las preguntas de opinión, el 98\% de los encuestados cree que el humo de su cigarrillo puede perjudicar a los fumadores pasivos y el $94 \%$ de la muestra opina que el humo de su cigarrillo puede molestar a los demás.

A la pregunta de si estarían de acuerdo en restringir los espacios para fumar a unas zonas concretas el $20 \%$ opina que no y el $80 \%$ que si.

Tabla 1. Cuestiones y resultados de la encuesta, expresados con un intervalo de confianza del $95 \%$.

\begin{tabular}{|l|c|c|c|c|}
\hline Cuestiones & & (n) & $\%$ & I.C. 95\% \\
\hline 1. ¿Sabes que en este espacio no se puede fumar? & $\mathbf{S i}$ & 39 & 78 & $(64,0-88,5)$ \\
& $\mathbf{N o}$ & 11 & 22 & $(11,5-36,0)$ \\
\hline 2. ¿Sabes que existen unos espacios destinados & $\mathbf{S i}$ & 42 & 84 & $(70,9-92,8)$ \\
a fumar? & $\mathbf{N o}$ & 8 & 16 & $(7,2-29,1)$ \\
\hline 3. ¿Sabes que la decisión de no fumar en este & $\mathbf{S i}$ & 11 & 22 & $(11,5-36,0)$ \\
edificio la han consensuado alumnos, profesores, & $\mathbf{N o}$ & 39 & 78 & $(64,0-88,5)$ \\
\hline y personal de adminstración y servicios? & & & & \\
\hline 4. ¿Crees que el humo del tabaco perjudica a los & $\mathbf{S i}$ & 49 & 98 & $(89,4-100,0)$ \\
fumadores pasivos? & $\mathbf{N o}$ & 01 & 02 & $(0,05-10,7)$ \\
\hline 5. ¿Crees que el humo del tabaco puede & $\mathbf{S i}$ & 47 & 94 & $(83,5-98,8)$ \\
molestar a los demás? & $\mathbf{N o}$ & 3 & 6 & $(1,3-16,6)$ \\
\hline 6. ¿Estás de acuerdo en restringir el fumar a & $\mathbf{S i}$ & 40 & 80 & $(66,3-90,0)$ \\
espacios concretos? & $\mathbf{N o}$ & 10 & 20 & $(10,0-33,7)$ \\
\hline
\end{tabular}


En la cuestión 7, planteada como "Puedes expresar tu opinión respecto a por qué no estás de acuerdo a restringir los espacios para fumar o sobre cualquier otro aspecto del edificio libre de humo de tabaco" se recababa la opinión abierta de quienes se negaban a que se restringieran los espacios o a aquéllos que querían expresar su punto de vista sobre el "edificio libre de humo de tabaco." Entre otras, se recogieron las siguientes opiniones:

Aún estando de acuerdo en restringir las zonas para fumadores, el $20 \%$ de los entrevistados dan su opinión al respecto: de éstas el $60 \%$ creen que "Es injusto que la medida haya sido tomada únicamente en un edificio y no en toda la universidad"; otras personas indican que "Faltan más zonas para fumadores" y otras que "Los espacios destinados a fumadores son inadecuados".

Existe un gran abanico de justificaciones para seguir fumando, tales como "España es una democracia y en un país libre puedo hacer lo quiera" (dicho sin animadversión y con pleno convencimiento); "Yo puedo hacer lo quiera..."; "Fumar en un sitio público no está prohibido..."; "Nadie me ha preguntado si quería el edificio libre de humo..."; "Todo el mundo fuma..."; "Somos más los que fumamos que los no fumadores..."; "Ahora mismo no sé por qué no quiero zonas restringidas..."; "No dejarme fumar donde quiera viola mis derechos..."; "Al ir a fumar a una zona permitida me siento discriminada y apestada..."; "No estoy de acuerdo porque no...."

\section{DISCUSIÓN}

Nuestra universidad no es una excepción en cuanto a que sus usuarios fumen en los espacios donde está prohibido hacerlo, baste para ello echar una ojeada a aeropuertos, cines, restaurantes, u otras universidades $^{14}$.

No cabe duda de que las personas entrevistadas están informadas de la prohibición de fumar en los espacios compartidos, ya que, de entrada el $78 \%$ lo reconocen así. Además, de los que en la cuestión 1 dicen "desconocer la prohibición", el 91\% responde a la cuestión 2 afirmando que conoce la existencia de "espacios destinados a fumadores" con lo cual se contradicen y "reconocen" que no se puede fumar en cualquier parte. Si computamos estas contradicciones el 98\% conoce la prohibición de fumar. Estas cifras contrastan con estudios realizados con estudiantes de secundaria que mostraban no tener una idea clara de dónde está permitido y no permitido fumar ${ }^{24}$. En nuestro caso tal vez los usuarios están mucho más concienciados, dada la intensa campaña de información y señalización llevada a cabo en el edificio.
Es muy significativo que el $78 \%$ de los encuestados dice no conocer el consenso para declarar el "edificio libre de humo" entre las distintas Facultades y Escuelas que constituyen la totalidad de usuarios de dicho edificio. Esto puede implicar una problemática más compleja: ¿son realmente representativos del colectivo, los alumnos que asisten como representantes a las juntas de centro? O bien ¿dichos representantes trasmiten a su colectivo las decisiones que se toman en estas juntas? Por otra parte, durante el mes anterior al pase de la encuesta, se han repartido más de mil dípticos, donde se informaba de cómo y cuándo el edificio había sido declarado libre de humo de tabaco, de los resultados de las votaciones en las distintas juntas de centro y del texto de la declaración. Sin embargo podría haber faltado la motivación para leerlo y en caso de hacerlo para tenerlo en cuenta.

Es destacable que la inmensa mayoría de entrevistados expresa su creencia de que el tabaco puede perjudicar y molestar a sus compañeros. Sin embargo, de ahí no se desprende una conducta respetuosa con los demás sino que -a sabiendas de que los perjudican y molestan- siguen fumando en los espacios compartidos. Esta opinión y actitud coinciden con las de la mayoría de estudios que las han analizado $25-28$.

Es más, una quinta parte de los que fuman en espacios compartidos afirma no estar de acuerdo en restringir las zonas para fumadores, es decir, desean que se institucionalice su derecho a fumar, aún a costa de perjudicar y molestar a los demás, porque como dice un sujeto, si tengo que ir a una zona "Me siento discriminada" o como dice otro "Siento que se violan mis derechos". Esta opinión coincide con la de otros estudios en que el hecho de que para fumar deban apartarse de los demás compañeros hace que los individuos se sientan marginados y estigmatizados ${ }^{22,29}$. En nuestro medio sigue valiendo el tópico de que "lo normal" es fumar en cualquier parte y lo excepcional es la prohibición de fumar ${ }^{20}$.

A pesar de que el $80 \%$ de los encuestados se declarara partidario de restringir los espacios en que se pueda fumar, mediante el lenguaje no verbal a menudo se mostraba una cierta reticencia. Esta actitud coincide con otros estudios en que cuando el fumador restringe su hábito, tiene la sensación de "estar haciendo un favor" a los no fumadores ${ }^{30}$. Por lo demás, entre las motivaciones que se dan para no restringir los espacios hay muy pocas que tengan una justificación ética. El clímax de desorientación (por llamarlo así) quizás lo alcancen aquellos sujetos que alegan que viven en un país democrático y que esto les permite hacer lo que quieran. Pocos argumentos se me ocurren para convencer a personas (universitarias) con una sensibilidad social tan atrofiada sobre la necesidad de respetar a los demás. Es una característica de las sociedades avanzadas obedecer las leyes actuando como autocensores y no por temor al castigo o a la 
fuerza bruta ${ }^{31}$. De todos modos el acto de fumar entre los jóvenes, a menudo ha sido considerado como un símbolo de identidad, rebeldía y atrevimiento ${ }^{32}$ alentado por la publicidad de la industria tabaquera que fomenta la creencia de que constituye valores como independencia, libertad, sentirse atractivos o controlar el estrés ${ }^{33}$. Por otra parte, cuando un grupo social intenta establecer unas normas o cambios que afectan a otro es habitual que se creen resistencias por parte de este último.

Otro argumento que aparece reiteradamente es el "Fumo porque no se ha prohibido en todos los edificios de la universidad". Si bien es cierto que no se ha explicitado la prohibición por las autoridades académicas, ésta ya existe a través del Real Decreto $192 / 88^{10}$, y en todo caso el incumplimiento de una ley por unos ciudadanos jamás puede justificar el incumplimiento de la misma por otros, con el agravante de insolidaridad, ya que en este edificio, el Consell Executiu (órgano de gestión compuesto por el rector y los vicerectores) eludiendo sus responsabilidades legales, en 2001 mandó someter a votación entre los usuarios del edificio jel cumplir o no una ley!. Afortunadamente resultó que una amplia mayoría era partidario de restringir el uso del tabaco a unos espacios concretos. Es tan notable como lamentable que este argumento "tan sólido" es el que en 2002 alegó la Junta de Facultad de Ciencias de la Universidad de las Islas Baleares para no restringir los espacios destinados a fumar en los edificios en que se impartían sus estudios.

En los comentarios se repite con cierta frecuencia un dato que indica una desinformación respecto a la prevalencia real de tabaquismo: "Todo el mundo fuma..."; "Somos más los que fumamos que los no fumadores.... En una encuesta realizada en 2002 en el edificio sólo 1 de cada 4 mujeres y 1 de cada 5 hombres son fumadores habituales ${ }^{25}$. Sin embargo entre los fumadores, parece muy extendida la creencia de que "todo el mundo fuma", tal vez para justificar su conducta diluyendo su responsabilidad personal en el medio social que irremediablemente les ha tocado vivir.

Un elevado número de entrevistados, a través del lenguaje no verbal, se mostraba tenso ante el hecho de tener que contestar con sinceridad que sabía que estaba prohibido fumar y que sabía que perjudicaba y molestaba a sus compañeros, sin embargo, ninguno reconoció su adicción a una droga que le llevaba a un comportamiento insolidario y antisocial. Tal vez la que más se acercó fue una chica entrevistada que en un corro que se formó comentó: "Yo no dejaré de fumar hasta que me den dos "hos...as" bien dadas.... Como puede deducirse, incluso reconociendo la adicción, se espera que la solución venga de fuera, porque internamente, a sus 22 años, muchos ya han tirado la toalla y se resignan a vivir encadenados al tabaco. A grandes rasgos, las causas alegadas para seguir fumando en espacios comunes coinciden con las de otro estudio cualitativo realizado en Canadá21: no saber que se están violando las reglas o que se molesta a los no fumadores y fumar como respuesta a la marginación y restricciones percibidas por los fumadores como irracionales. Una diferencia notable -tal vez porque nuestra encuesta está dirigida a jóvenes y la otra a adultos en general- es que en nuestro trabajo, nadie alega la adicción/dependencia como motivo para fumar en espacios compartidos. No obstante en la mayoría de encuestas vemos que más de un $60 \%$ de fumadores desean abandonar el tabaquismo y no pueden ${ }^{25,34}$.

Los resultados de esta encuesta servirán de base a un trabajo posterior, elaborando un estudio cualitativo del por qué conociendo la prohibición de fumar y los efectos perjudiciales y molestos del tabaco para uno mismo y para los demás, no se respetan los lugares de convivencia. Creemos que la combinación de técnicas cuantitativas y cualitativas enriquecerá los resultados $^{35}$. Tal vez a partir de éstos, las autoridades académicas y profesorado se sientan más concienciados y responsabilizados en el formar universitarios no solamente en conocimientos sino también en actitudes que puedan constituir un modelo social cuanto menos saludable y en todo caso basado en el respeto a los demás.

\section{REFERENCIAS}

1. U.S. Department of Health and Human Services. Smoking and Health: Report of the Advisory Committee to the Surgeon General of the Public Health Service. Rockville, Maryland, USA. Office on Smoking and Health Centers of Disease Control, Public Health Service; 1964. Disponible en: http://www.cdc.gov/ tobacco/sgr/sgr_ 1964/sgr64.htm [consultado el 11/02/04].

2. U.S. Environmental Protection Agency. Respiratory Health effects of passive smoking: lung cancer and other disorders. Washington DC: Office of Research and Development. Office of Health and Environmental Assessment, 1992. EPA/600/6-90/006F.

3. Muggli ME, Hurt RD, Repace J. The tobacco industry's political efforts to derail the EPA report on ETS. Am J Prev Med 2004; 26: 167-77.

4. U.S. Environmental Protection Agency (EPA) [comunicado de prensa 07/01/93]. EPA dessignates passive smoking a "Class A" or Known Human Carcinogen. Disponible en: http://www.epa.gov/history/topics/smoke/01.htm [consultado el 24/02/04].

5. Edler F, Zeeman G. Riesgos para la salud derivados del consumo voluntario e involuntario de tabaco. Rev Esp Salud Pública 2003; 77: 11-36.

6. 56 World Health Assembly WHO Framework Convention on Tobacco Control. Document A56-8 Geneva: WHO, 2003. 
7. González J. El Convenio Marco de la Organización Mundial de la Salud para el Control del Tabaco y el Plan Nacional de Prevención y Control del Tabaquismo. Prev Tab 2003; 5: 76-9.

8. Declaración de la Conferencia de Berlín [editorial]. Rev Esp Salud Pública 2003; 77: 7-9.

9. Ley General de Sanidad de 14/1986, de 25 de abril. BOE $n^{\circ} 102$ de 29 de abril de 1986 .

10. Real Decreto 192/1988, de 4 de marzo, sobre limitaciones en la venta y uso del tabaco para la protección de la salud de la población. BOE $n^{\circ} 59$ de 9 de marzo de 1988.

11. Ministerio de Sanidad y Consumo. Plan Nacional de Prevención y Control del Tabaquismo, 2003-2007. Rev Esp Salud Pública 2003; 77: 441-73.

12. Sasco AJ, Mélihan-Cheinin P, d'Harcourt D. Legislación sobre el consumo de tabaco en el ámbito laboral y en los espacios públicos de la Unión Europea. Rev Esp Salud Pública 2003; 77: 37-33.

13. Villalbí JR. III Congreso Nacional sobre Prevención y Tratamiento del Tabaquismo [conclusiones del congreso]. Zaragoza 2003. Diponible en: http://www. faecap. com/Documentos/Documentos_Jornadas_ y_Congresos/conclusiones_del_iii_congreso_na.htm [consultado el 03/02/04].

14. López V. Se fuma en la mitad de los lugares donde está prohibido hacerlo. Consumer 2003; 63: 3-9.

15. Ayesta FJ, de la Rosa L. ¿Por qué fuman más hoy en día las mujeres?. Prev Tab 2001; 3: 155-60.

16. Di Franza JR. RJR: El camello de la comiquita de Nabisco promociona los cigarrillos Camel entre los niños. J Am Med Assoc 266, 3149-53.

17. Altman DG. Promoción tabáquica y susceptibilidad al uso del tabaco en adolescentes de 12 a 17 años en prueba nacionalmente representativa. Am J Public Health 1996; 86: 1590-3.

18. Nelson E, White D. La concienciación infantil de la publicidad de cigarrillos en televisión. Health Education J 1992; 51: 34-7.

19. Willemsen MC, De-Zwart WM. La efectividad de las estrategias y políticas educativas para reducir el fumado de adolescentes: Un análisis de la evidencia. J Adolesc 1999; 22: 587-99.

20. Becoña E. Bases teóricas que sustentan los programas de prevención de drogas. Madrid 1999. Plan Nacional sobre Drogas. Ministerio de Interior. p. 235-45. Disponible en: http://www.mir.es/pnd/publica/pdf/bases.pdf [consultado el 04/02/04].

21. Polland BD. The "considerate" smoker in public space: the micro-politics and political economy of "doing the right thing". Healh \& Place 2000; 6: 1-14.
22. Polland BD. Smoking, Stigma, and the Purification of Public Space. En: Kearns RA, Gesler WM editores. Putting Health Into Place: Landscape, Identity and WellBeing. Syracuse, NY, Syracuse University Press; 1998. 208-25.

23. Pericàs J. Propuesta de Constitución del Edificio Guillem Cifre de Colonya como "Libre de humo de tabaco". Enfermería Científica 2002; 240-241: 41-6.

24. Ballestin M, Bosch N, Nebot M. Conocimiento y aplicación de las normas sobre tabaquismo en centros de enseñanza secundaria. Aten Primaria 1999; 24: 589-93.

25. Pericàs J, Bauzà M LL, Ponsell E. Epidemiología del tabaquismo en la Universitat de les Illes Balears [en prensa]. Prevención del Tabaquismo.

26. Franco JA, Pérez A, García A, Marrón R, Clemente ML, Rubio E. ¿Qué conocemos y qué actitud se puede tomar acerca del consumo de tabaco en la educación no formal? Arch Bronconeumol 2004; 40: 10-6.

27. Villalbí JR, Nebot M, Ballestín M. Los adolescentes ante las sustancias adictivas: tabaco, alcohol y drogas no institucionalizadas. Med Clin (Barc) 1995; 104: 784-8.

28. Pérez-Trullén A, Clemente R, Marrón R. La intervención antitabáquica del médico en la escuela: dilema entre voluntariado versus gabinete técnico. Prev Tab 2001; 3: 46-50.

29. Parry O, Platt S. Smokers at risk: implications of an institutionally bordered risk-reduced environment. Health \& Place 2000; 6: 117-23.

30. Bull SB, Pederson LL, Ashley MJ. Relationship of smoking status to changes in knowledge and attitudes concerning restrictions on smoking. Can J Public Health 1994; 85: 103-5.

31. Foucault M. Power/Knowledge -Selected Interviews and Other Writings. Hertfordshire, UK: Harvester Wheatsheaf; 1980.

32. Robbins MC, Kline A. To Smoke or Not to Smoke: A Decision Theory Perspective. Soc Sci Med 1991; 33: 1343-7.

33. Galván C, Díaz NM, Suárez RG. Exposición al tabaco y patología respiratoria en la infancia. Rev Esp Pediatr 1999; 55: 323-7.

34. Garcia V, Ramos M, Hernan M, Gea T. Consumo de tabaco y hábitos de salud en los adolescentes de Institutos de Enseñanza Secundaria Pública en Granada. Prev Tab 2001; 3: 215-23.

35. March JC, Prieto MA, Hernán M, Solas O. Técnicas cualitativas para la investigación en Salud Pública y gestión de servicios de salud: algo más que otro tipo de técnicas. Gac Sanit 1999; 13: 312-9. 